\title{
Cyclical menouria syndrome following mid trimester abortion: a case report
}

\author{
Pratiksha Gupta, Anju Huria, Reeti Mehra, Usha Dalal \\ Government Medical College and Hospital, \\ Chandigarh India
}

\begin{abstract}
Menouia is terminology used for the passage of menstrual blood only in the urine and results from supraisthmic uterovesical fistula. Report here, for the first time is a case of cyclical menouria, that developed after induced mid trimester abortion in a $26 \mathrm{yrs}, \mathrm{P}_{2} \mathrm{~L}_{2} \mathrm{~A}_{1}$ which was diagnosed relatively with ease from history, simple selected investigations who underwent successful surgical repair meaning complete resolution of cyclic haematuria.
\end{abstract}

Key words- Menouria; uterovesical fistula; induced abortion

\section{Introduction}

Passage of menstrual blood only in the urine, due to supra-isthmic uterovesical fistula is called menouria. ${ }^{1}$ Uterovesical fistulas belongs to the least common types of urogenital fistulas. They are mostly caused by repeated caesarean sections, which are increasing, and their complications can be expected to increase as well. ${ }^{2}$ other causes include singular reports on dilatation and curettage for induced or incomplete abortion, forceps use, and other causes. Here by we report a case of type 1 vesico uterine fistula developing after induced abortion which is rare and the management protocol is discussed with specific simple diagnostic modalities, and definite surgical approach.

\section{Case report}

A 26 years old woman presented to Department of Gynaecology and Obstetrics with the history of amenorrhea and cyclical menouria since $2 y$ rs but with complete urinary continence. She had two full term normal vaginal delivery in hospital without any history of prolonged, obstructed or difficult delivery or any other complications. There is a history of induced abortion which was done 2 yrs back, at 4 months of gestational age, it was done in a hospital, according to the patient dilatation and evacuation was done by some instrument, and the foetus was removed in pieces. This procedure took approximately 2 hours, she had increased blood loss with incapacitating pain and so she was admitted in that hospital for few days. In the next cycle she had amenorrhea, but started having cyclical haematuria, without any history of urinary incontinence. General systemic examination was within normal limits, per abdomen examination was soft, and no mass was palpable. Obstetrical examination revealed cervix and vagina to be healthy, no urinary leak or fistula could be identified. Per vaginal examination revealed uterus to be retroverted, normal sized and adnexa was normal. To confirm the diagnosis the patient was subjected to simple specific investigations. Ultrasound examination showed a communication between bladder and supracervical region (Figure1). Methylene blue dye test was done, the urine turned blue as was visualized in the catheter which was inserted per urethra prior to the dye instillation. Methylene blue dye was injected through a transcervical catheter (Figure 2). Patient was subjected to cystoscopic examination; cystoscopy revealed a fistula of $3 \mathrm{~cm}$ x $3 \mathrm{~cm}$ size which was lying transversely above the trigone with ragged oedematous angry looking margins, granulation tissue was seen within the fistulous tract, which bled on touch. A final diagnosis of uterovesical fistula was made and patient was planned for transabdominal repair. Per operatively uterus and adnexa were of normal size, omentum was

Correspondence

Dr Pratiksha Gupta, M D; Asst. Professor

Department of obstetrics and gynecology, Government Medical College and Hospital,

Sector 32 B, Chandigarh India -160030,

Email-drpratiksha@gmail.com 


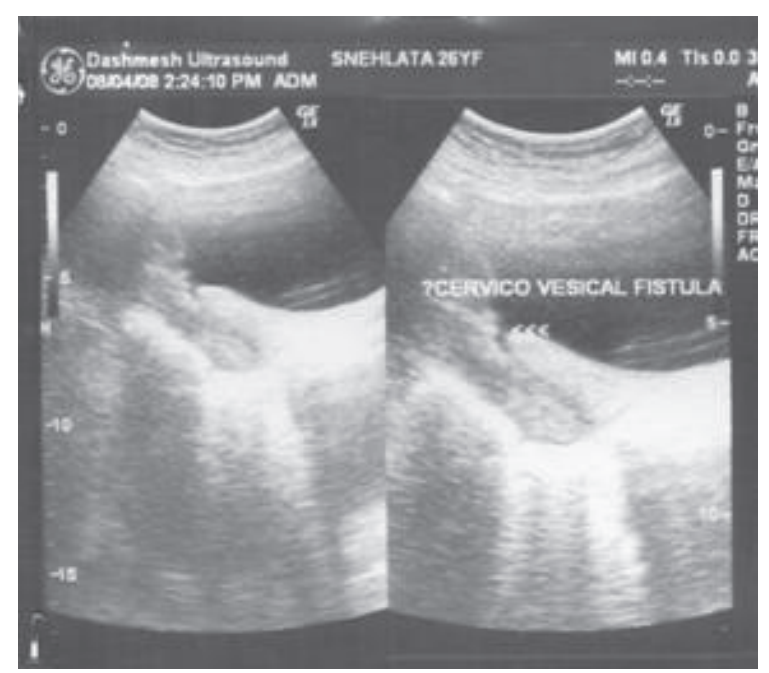

Figure 1. Ultrasound picture showing communication between urinary bladder and uterus.

adherent to bladder, part of dome of bladder was seen invaginating between the uterine musculature. Sharp dissection of uterovesical fold of peritoneum was done. On more dissection, bladder was seen communicating with the opening in body of uterus which was just above the internal cervical os, this defect was $2 \mathrm{~cm}$ in size; a $3 \times 3 \mathrm{~cm}$ size rent was seen in posterior wall of the bladder (Figure3). The fistula tract was excised sharply from the uterus and bladder, The uterus was repaired in a single layer by No.1 polyglycolic acid suture. The bladder was repaired in two layers in a running fashion with number 3-0 polyglycolic acid suture. Bladder peritoneum was raised as a flap and the bladder incision line was reinforced with this flap. Urethral catheter was left behind. The woman was discharged on post operative day 10 with no urinary leakage, menouria or urinary tract infection.

\section{Comment}

Although uncommon, vesico-uterine fistula cannot be considered a rarity in view of more than 800 published cases in the literature. Three types of vesicouterine fistula have been described-Type1 is characterized by the following symptoms: menorrhea, here the term is understood as lack of vaginal menstruation, cyclical appearance of blood-stained urine during the expected time for menstruation, and complete continence of urine. This triad has been known as Youssef's Syndrome. Type2 - vesicouterine fistula is associated with the coexistence of menouria, vaginal menses, and constant or periodic incontinence of urine. Type3-is characterized by the lack of menouria, normal vaginal menstruation, and constant or periodic incontinence of urine. ${ }^{3}$ Most patients present early postoperatively, while others are seen months later with urinary incontinence. Recurrent urinary tract infections, menstruation through the bladder (menouria), secondary infertility and amenorrhea are less common reasons for consultation ${ }^{4}$ Type 1 is unusual among urogenital fistula because of urinary continence. Symptoms are well tolerated by the patient since there is no leakage of urine. For reasons unclear to date, at the level of internal os a stop in passage occurs, sphincter mechanism of the uterine isthmus and with reference to normal pressure gradient that exists could explain menouria and absence of vaginal bleeding or urinary leaks. Since presence of urine in genital tract decreases the reproductive capacity, so type 1 fistula patients may seek medical assistance because of their wish to have children and not because of urinary symptoms, as in our case. With patient history and selected investigations diagnosis is relatively easy. The surgical repair of these fistulae is the standard treatment, especially with delayed fistulae, with achievement of total continence, and complete resolution of cyclic haematuria. Meticulous practice of obstetric and surgical principles during caesarean section and other obstetrical intervention can prevent the formation of these fistulae. The direct or indirect causes of vesicouterine fistulas identified are diverse. They include singular reports on dilatation and curettage for induced or incomplete abortion, forceps use, external cephalic version, degenerating myoma adherent to the bladder, craniotomy, septicemia, ruptured uterus, manual removal of placenta, pelvic tuberculosis, and intrauterine device perforation, the remainder being connected with cesarean section. ${ }^{5}$ As already stated very limited case reports are there to attribute it to abortion. Spontaneous abortion through the bladder has been reported by S. Guruvarein in 2004. ${ }^{6}$ In 1978 Barton reported a case of vesico uterine fistula after elective abortion. ${ }^{7}$ Obstetric vesicovaginal fistula is a major cause of urinary incontinence in lowincome countries, with an estimated 130000 new cases annually. ${ }^{8}$ Simple diagnostic tests are required for confirming the diagnosis-proper history, per speculum examination, methylene blue dye test, cystoscopy, hysterogram, hysterosalpingogram and ultrasound examination. Intravenous pyelogram can be done, if still a definitive diagnosis is not established. Successful fistula repair involves closure of the fistula, and careful postoperative care, the most important aspect of which is continuous bladder drainage to allow tension-free healing. The reported success rate of fistula closure at one study was $92 \%,{ }^{9}$ with immediate residual incontinence occurring in $33 \%$ of women. The duration of catheterization after surgery is traditionally about 14 days, with extension up to 21 days if the repair involves urethral reconstruction, extensive bladder neck surgery, or if postoperative wound breakdown has occurred..$^{10}$ In our case catheterization was done for 10 days with good results. Conservative management could be tried, if discovered soon after delivery or abortion, indwelling catheter can be kept 


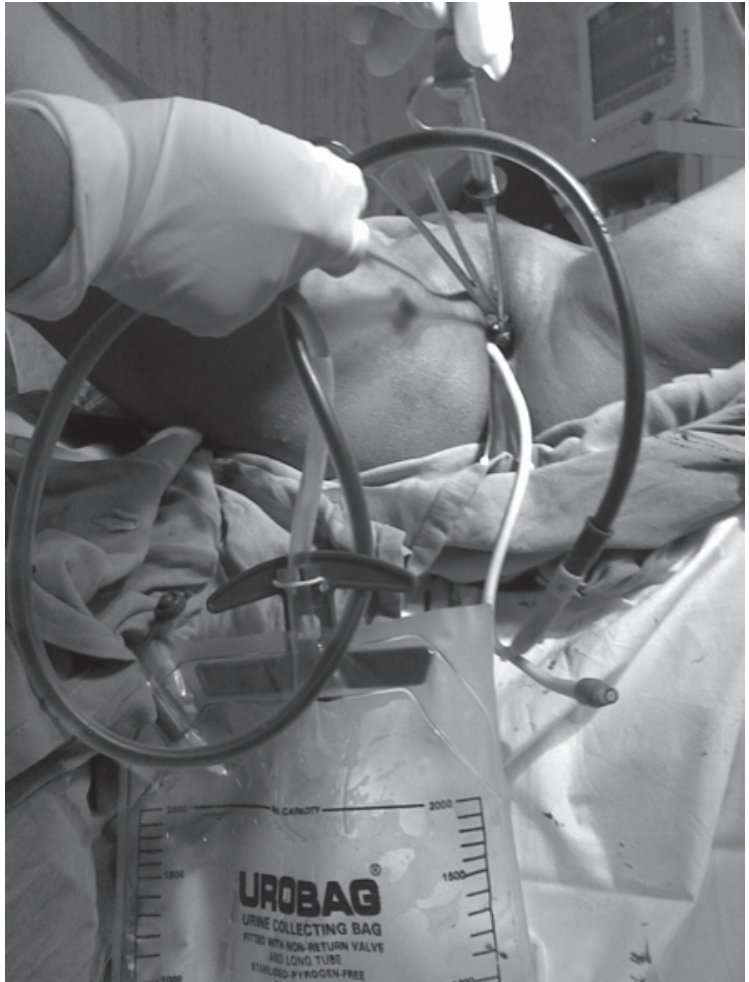

Figure 2. Methylene blue dye test (urine stains blue).

for 4-8 weeks, $5 \%$ cases reported to have spontaneous closure, hormonal treatment could be tried in Youssuf syndrome with very small fistula. Reported pregnancy rate after repair is $31.25 \%$, and term delivery rate is $25 \%$, and surgery is the mainstay and transperitoneal route is most effective. After repair hysterectomy is not required for treatment of vesicouterine and fertility can be preserved in selected patients. Very few laparoscopic repairs have also been described. The gynecologist must be aware of the possibility of causing this injury. Even rupture of a full bladder during an extraction of a baby's head in the course of a cesarean section can cause vesicouterine fistula. This instance demonstrates that not only inadequate surgical technique can lead to the lesion's formation but that careful bladder emptying before operation is essential. Meticulous practice of obstetric and surgical principles during obstetrical procedures can prevent the formation of these fistulas.

\section{References}

1. Parulekar SV. Lochiauria: a new entity- A case report. J Postgrad Med 1989 Oct; 35(4):228-9.

2. Lent V, Laaser M. Uterovesical fistula. Aktuelle

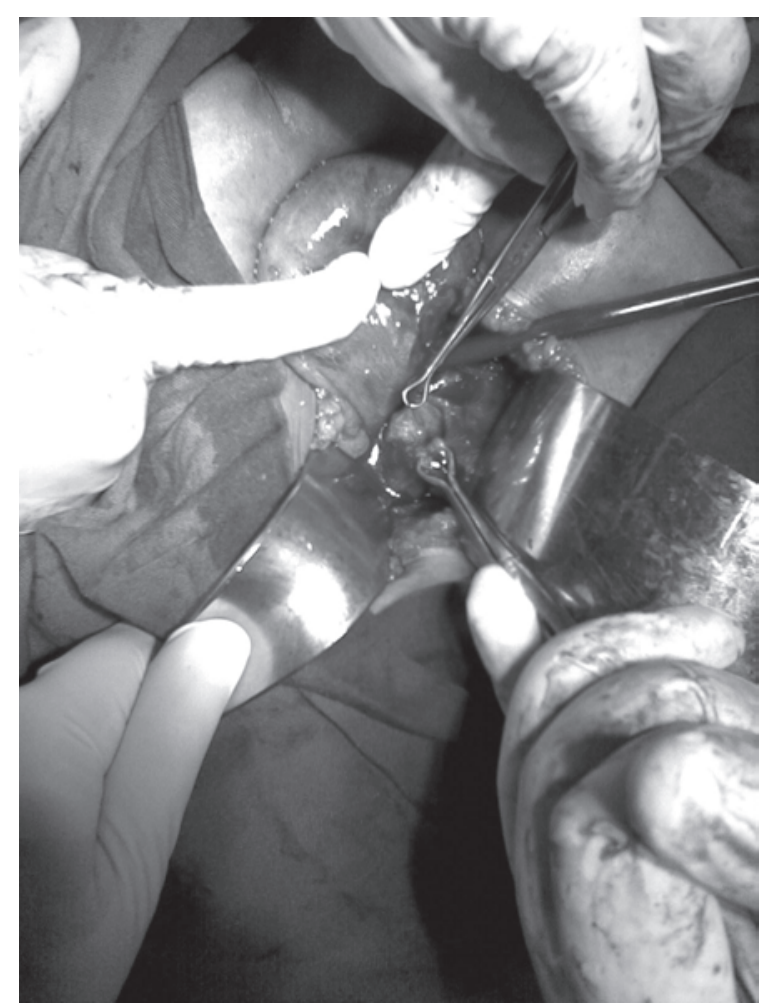

Figure 3. Surgical dissection shows urinary bladder and uterine opening separately.

Urol 2005 Feb; 36(1):61-3; quiz 65-6.

3. Maciej J'ózwik, Marcin J'ózwikU. Clinical classification of vesicouterine fistula. International Journal of Gynecology \& Obstetrics 2000; 70: 353-357.

4. Lenkovsky Z, Pode D, Shapiro A, Caine M. Vesicouterine fistula: a rare complication of cesarean section. J Urol.1988 Jan; 139(1):123-5.

5. Taxer ML. Vesicouterine fistula - a review. Obstet Gynecol Sure 1986; 41: 743-753.

6. S. Guruvarea, P. Kushtagia, J. Thomasb Spontaneous abortion through the bladder, International Journal of Gynecology and Obstetrics 2004; 87:172-17.

7. Barton, J. J., Grier, E. A. and Mutchnik, D. L. Ureterouterine fistula as a complication of elective abortion. Obst Gynec. Suppl 1978; 52: 81S.

8. Wall LL. Obstetric vesicovaginal fistula as an international public-health problem. Lancet 2006; 368(9524):1201-9[2].

9. Muleta M. Obstetric fistulae: a retrospective study of 1210 cases at the Addis Ababa Fistula Hospital. J Obstet Gyneacol 1997; 17(1):68-70.

10. Nardos R, et al, Duration of bladder catheterization after surgery for obstetric fistula, Int J Gynecol Obstet (2008), doi:10.1016/ j.ijgo.2008.05.021. 